\title{
Correlation of CAT scan and visual field defects in vascular lesions of the posterior visual pathways
}

\author{
D. L. M c A ULEY A N D R . R OSS R USSELL \\ From the Institute of Neurology, The National Hospital, Queen Square, London
}

SUMMARY Thirty-nine patients with various types of isolated homonymous hemianopias resulting from ischaemic lesions in the posterior parts of the cerebral hemisphere were examined by CAT scanning. Most had localised low density lesions within the distribution of the posterior cerebral artery. The location of these lesions (deduced from a separate anatomical study of postmortem brain cut in the plane of the CAT scanner) was correlated with visual field defects. Lesions giving rise to quadrantic defects were smaller than those causing total hemianopias; lower quadrantic defects tended to occur in superior cuts and vice versa. Macular sparing was associated with survival of the occipital pole in some instances. Bilateral cases had a higher prevalence of associated defects.

Computerised tomography (CAT) has led to a reappraisal of many aspects of cerebrovascular disease. It is now possible in many cases to delineate the site and extent of cerebral infarction, to study its natural progression, and to exclude other types of lesion such as haemorrhage or tumour.

Because of the frequency of isolated ischaemic lesions of the visual pathways and visual cortex a unique opportunity exists to relate the extent of the visual loss to the site of the infarct in the light of knowledge already available from the classical studies on missile injuries by Holmes (1918), Henschen (1926), and later by Spalding (1952).

Preliminary findings of the present study with a smaller number of patients have already been published (McAuley and Ross Russell, 1977).

\section{Anatomy}

The primary visual cortex in man, Brodman's area 17 , is situated almost entirely on the medial aspect of the occipital lobe, a small amount extending onto the posteromedial aspect of the occipital pole. The extent and symmetry of the visual cortex is extremely variable (Polyak, 1957).

\footnotetext{
Address for reprint requests: Dr R. W. Ross Russell, Institute of Neurology, The National Hospital, Queen Square, London WC1N 3BG.

Accepted 2 October 1978
}

The central $8-10^{\circ}$ of vision projects to the posterior pole while the serial concentric zones of the retina from the macula to the periphery are represented from behind forwards, above and below the calcarine fissure (Spalding, 1952). The fissure runs upwards and forwards from the occipital pole. In Fig. 1, taken from Holmes (1918), the lips of the calcarine fissure have been opened out to show the floor. Corresponding parts of the visual field and cortex have been marked in the same way. Much of the cortex, particularly that representing the horizontal octants, is buried within the calcarine fissure.

A section in the usual operating plane of the scanner (see below) parallel to the orbitomeatal line would in higher cuts tend to show the more anterior visual cortex-that is, the more peripheral visual field especially the vertical octantswhile the lowest cut passing through the cerebral hemispheres would tend to display the occipital poles representing the central portion of the visual fields. Intermediate cuts would demonstrate cortex representing the peripheral fields, with the vertical octants situated medially and the horizontal octants laterally. Higher slices would tend to display cortex above the fissure, representing the lower half field, and vice versa. Figure 2 shows diagramatically the usual plane of the EMI scanner. Approximately three or four slices cut through the visual cortex, the lowest slice including mainly the posterior pole. 

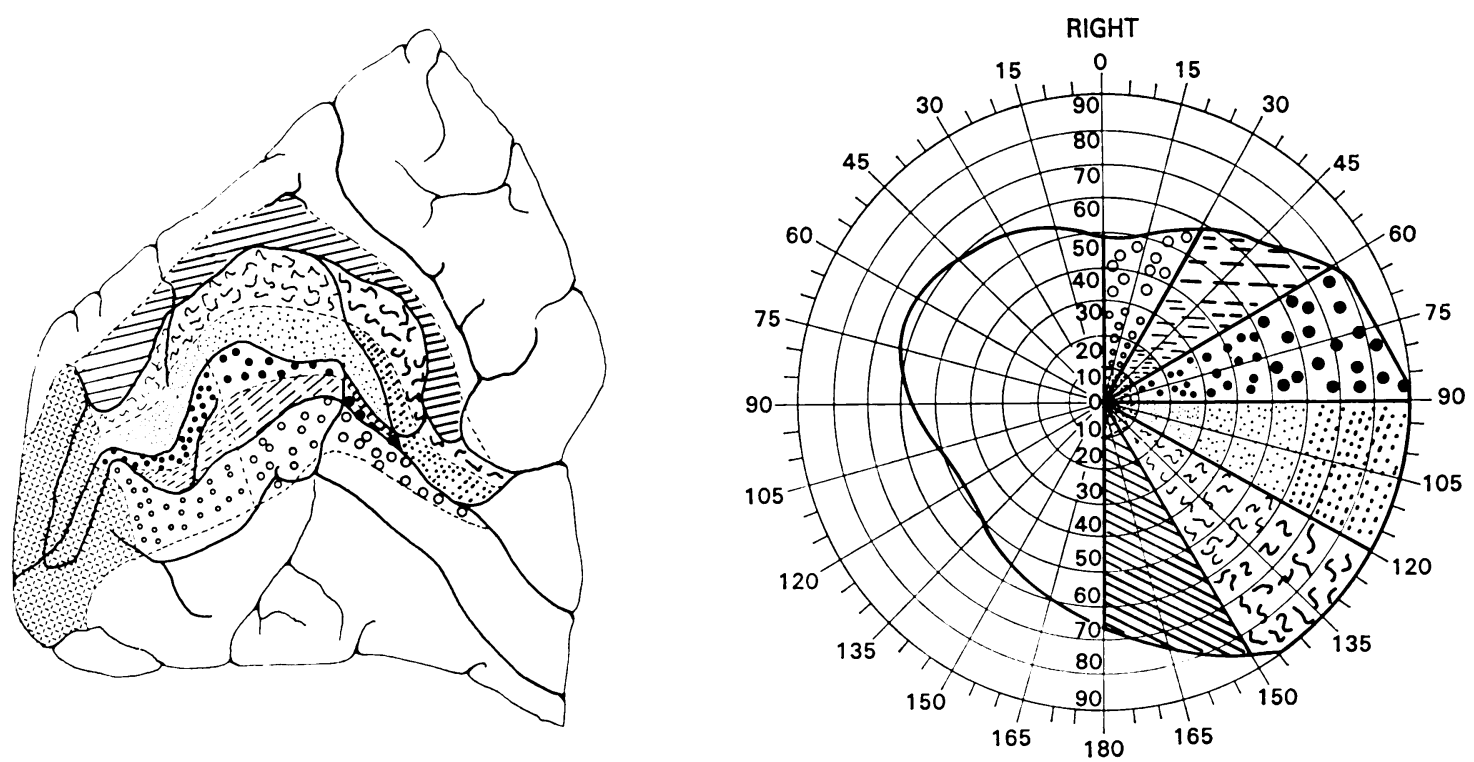

Fig. 1 Illustration from Holmes (1918) showing the representation of the visual field on the primary visual cortex with corresponding areas marked in the same manner. The lips of the calcarine fissure have been spread apart to demonstrate the floor.

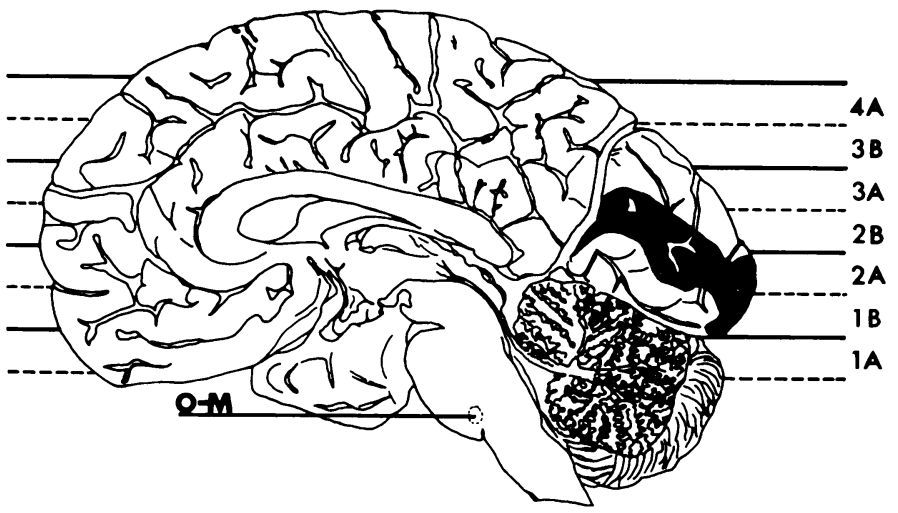

Fig. 2 Schematic diagram of the planes of the CAT scanner. Primary visual cortex shown in black.

\section{Vascular supply}

Figure 3 contains two illustrations taken from the original description of cerebral arterial territories by Beevor (1909) showing ascending horizontal slices (and, therefore, not strictly in the plane of the scanner) numbered 1 to 5 . The contribution of the posterior cerebral artery (P) is greatest in the lowest slice and diminishes with ascending slices as the middle cerebral arterial contribution (M) increases. The posterior cerebral artery territory normally includes all the striate cortex. The posterior pole lies close to the border zone between middle and posterior cerebral artery territories. A more elaborate demonstration of the arterial supply in horizontal slices can be found in the work of Salamon (1973) and Salamon et al. (1977).

\section{Methods}

To study the representation of the visual cortex in separate CAT scan slices, eight human brains were cut in $13 \mathrm{~mm}$ slices (the standard width of the EMI scan slice) in the usual operating plane of the scanner. At the National Hospital the scanner is operated in a plane parallel to the orbitomeatal line-that is, approximately 10-15 degrees to the horizontal plane or Reid's baseline (Gawler et al., 1975). The effect of altering this plane on the 


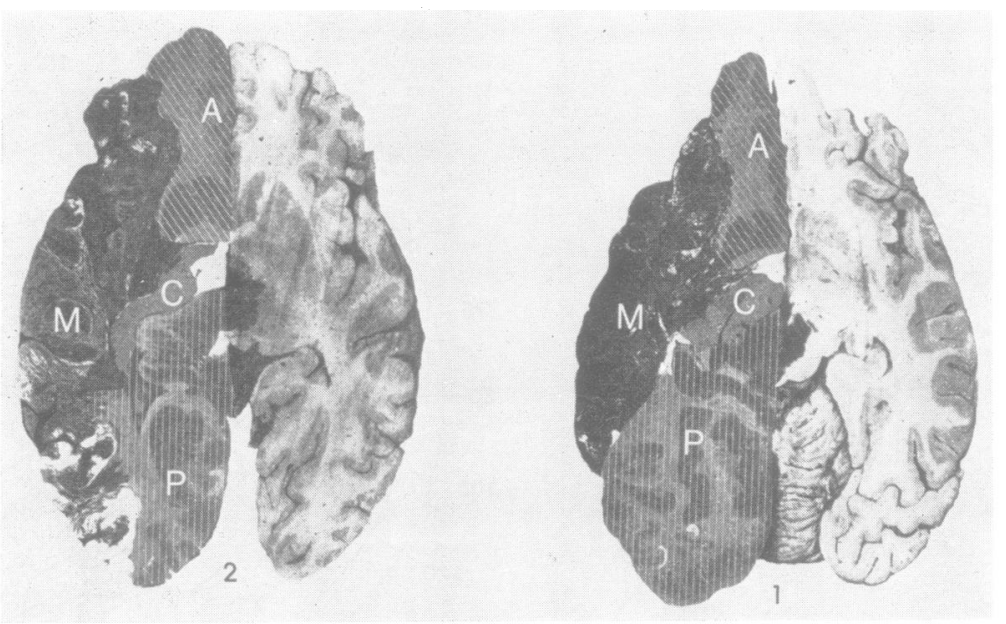

(a)
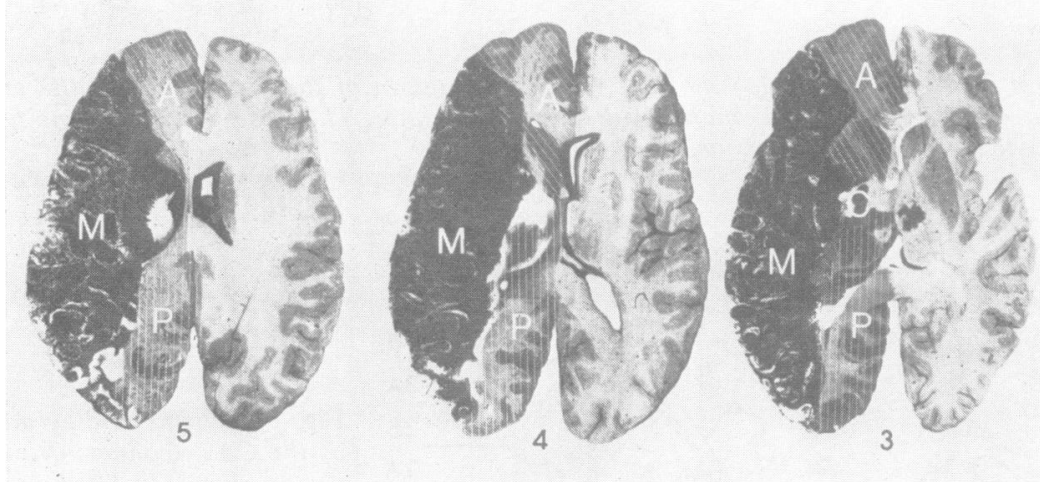

(b)

Fig. 3 Illustrations taken from Beevor (1909) showing the vascular territories in ascending horizontal slices. $A=$ anterior cerebral artery, $M=$ middle cerebral artery, $P=$ posterior cerebral artery, $C=$ choroidal artery.

structures demonstrated in any one slice can be considerable but has been studied in detail by Roberts et al. (1977). In the present study particular care was taken to orientate the brain correctly. The brains were fixed in formalin in the usual way by suspension from the basilar artery. While this does not result in any distortion of the cerebral hemisphere shape, the relationship of the brainstem to the hemispheres is altered. For this reason the brains were cut upside down on a purpose-built jig which enabled fixed landmarks, determined by the study of a series of normal skull radiographs and pneumoencephalograms, to be used to establish the correct alignment for the initial cut. Thereafter serial slices could readily be cut using a brain slicing box. The slices were then stained with a copper sulphate-phenol-ferrocyanide method (Roberts and Hanaway, 1969). This method stains the grey matter, leaving the white matter unstained, and facilitates macroscopic study by increasing the contrast. The extent of the visual cortex in each slice could easily be determined under low power magnification by observation of the unstained stria of Genari. The visual cortex was marked in on the superior surface of each slice using black marking ink and then photographed. Tracings were made from enlarged photographic prints and slices at the same level were assembled to show the amount of variation.

Computerised tomography scans from 39 patients suffering from a variety of isolated visual field and other visual defects secondary to 
ischaemic lesions were examined retrospectively. Only those patients in whom the visual field defect had been plotted in detail were included. Visual fields were plotted kinetically on a Goldman perimeter with varying target sizes. A number of the field examinations were rechecked by one of the authors (DLM), and tangent screen examinations were performed where appropriate.

The patients were divided into four types of visual field disorder: quadrantanopias, complete hemianopias, scotomatous defects, and bilateral defects. The interval between onset of illness and scan varied from 14 days to 20 years. The standard $13 \mathrm{~mm}$ cone (EMI CAT scanner) was used with centering heights of 30,55 , and $80 \mathrm{~mm}$ above the orbitomeatal line. The scans were compared with the "normal" scan obtained by this method (Gawler et al., 1975). Variations from the orbitomeatal scanning plane, undetected at the time of the x-ray examination, were excluded by comparison with the illustrations from Roberts et al. (1977). The criteria of infarct size were based on the limits of the low density area checked on the printout if necessary. Not all patients were given intravenous enhancement and the additional features obtained with this technique were not used in assessment. The details of infarct evolution were not studied, most patients having been scanned only once as near to the time of the campimetry as possible. An important normal variation which had to be considered when interpreting the scans was the size of the ventricular occipital horn. This extends a long way back into the occipital lobe and, if it is asymmetrical or the head positioning is oblique, the ventricle can be confused with an infarct. The distribution of the infarct in each scan slice was noted with reference to the slice containing the pineal gland ( $P$ slice, see later). The number of slices involved, the distribution of the involved slices, and the state of the occipital pole were all noted. Other radiological features such as the presence of cerebral atrophy and unsuspected infarcts in other areas were recorded.

\section{Results}

PATHOLOGICAL STUdies

In the cadaver brain slices (illustrated in Figs. 4-7) the visual cortex shows up in at least two slices in each brain and in one brain in as many as four slices. There is considerable variation between brains. This method of mapping of the visual cortex shows only the superior surface of the cadaver brain slice while in the CAT scan slice the structures displayed are the "average" of the $\mathrm{x}$-ray transmission densities throughout the thickness of the scan slice in each theoretical "cell" (see Gawler et al., 1975). The difference is apparent when cadaver slices are viewed from both the superior and inferior surfaces.

The major visual cortex representation occurs in the slice which included the pineal gland (Fig. 8). when this cadaver slice is viewed from the medial aspect it contains the longest part of the calcarine fissure. Considerable variation in the anatomical features demonstrated in any one scan slice (Ia, Ib, $\mathrm{IIa}$, and so on) is found in different patients, depending on such factors as positioning and shape of the patient's head and the centering heights.

\section{PATIENTS WITH VASCULAR HEMIANOPIA}

The scan slice most consistently showing infarction is that in which the pineal gland is visualised and a system of using the pineal (P) slice as the reference has been adopted. Slices below the pineal gland are called $P-1, P-2$, and so on, and those above $P+1, P+2$, and so on in serial order. With this notation the $P-1$ slice could be expected to contain mostly macular cortex, while the $P$ slice some, mainly upper, field and $P+1$ little if any macular (mainly peripheral) field.

The aetiology of the vascular lesions in this series of patients was limited to migraine (M), cerebral emboli (CE) or cerebrovascular disease (CVD).

Table 1 gives the sex and age of the patients together with the aetiology, the duration of infarct, and the visual field defect. The type of field defect related to the number of involved slices is shown in Table 2. Figures 9-14 illustrate some of the findings in the patients studied.

Three patients had completely normal scans and two others had equivocal areas of low density in the appropriate region, one to five months after the onset of their illness. One of those with a normal scan surprisingly had a complete hemianopia but with macular sparing, while the other two had an upper quadrantanopia and a homonymous scotomatous defect respectively. Of the two with equivocal low density occipital lesions, one patient had a small paracentral homonymous scotoma and the other a partial homonymous inferior quadrantanopia.

Conversely, there were two patients who had unsuspected infarcts in the occipital lobe without corresponding visual field defects. Both were small and restricted to one scan slice-one was very high in the $\mathbf{P}+3$ slice and probably did not involve the striate cortex, while the other at the level of $\mathbf{P}+1$ probably involved the posterior optic radiation rather than cortex. 

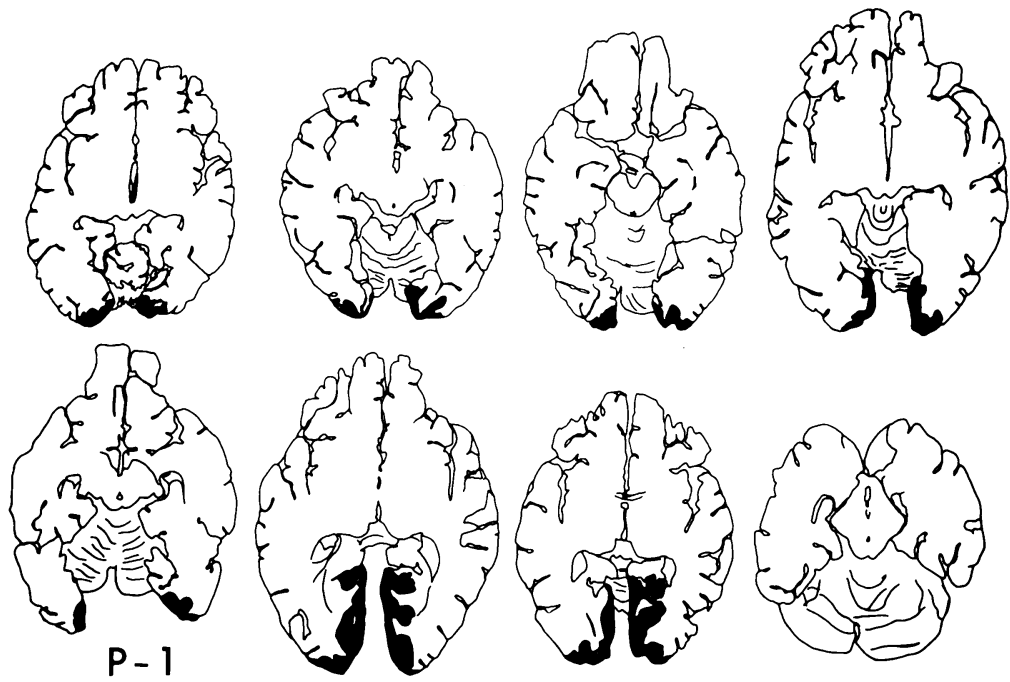

Fig. 4

Figs. 4-7 Tracings of brain slices with extent of visual cortex marked in black at each level in each of eight brains. $P=$ pineal slice, $P-1 ; P+1$ and $P+2$ above and below pineal slice respectively (see text).
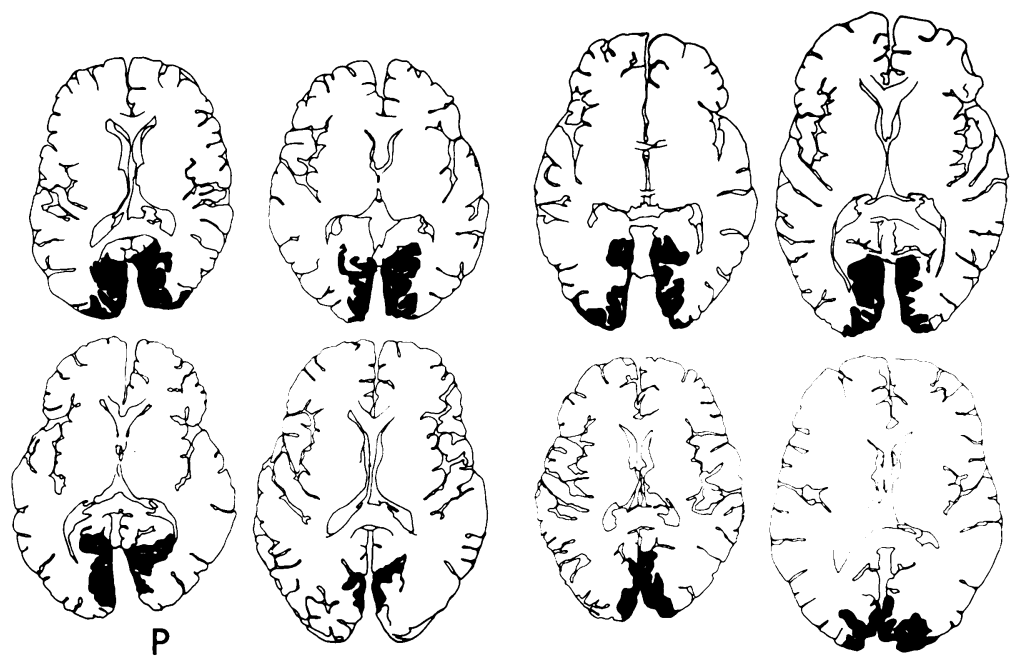

Fig. 5 


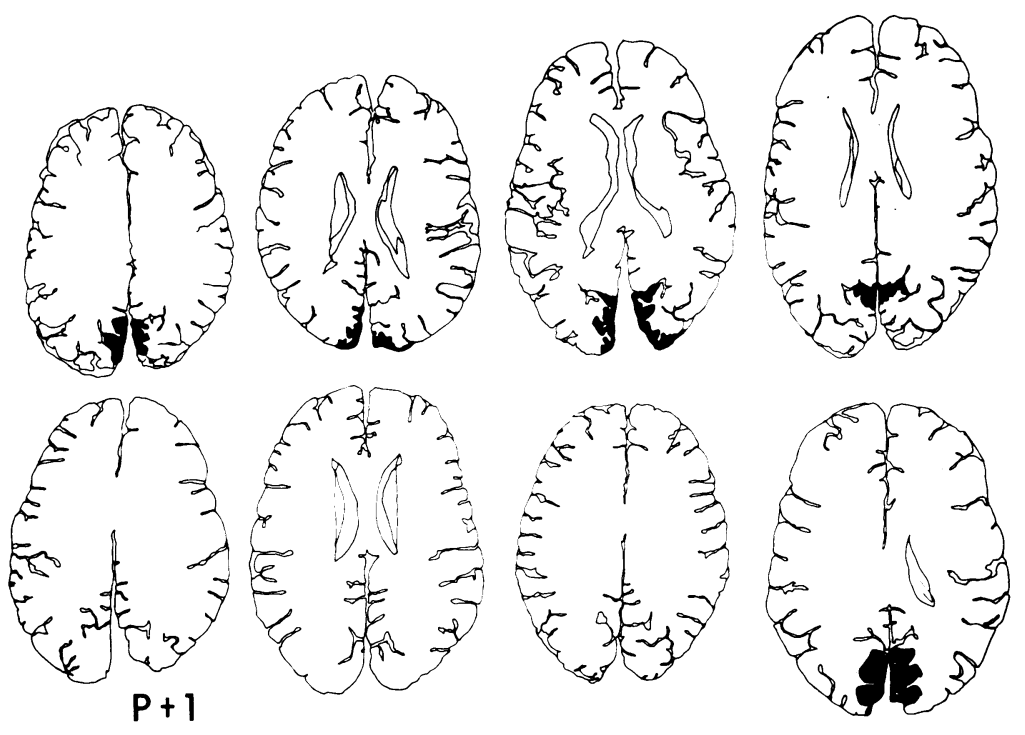

Fig. 6

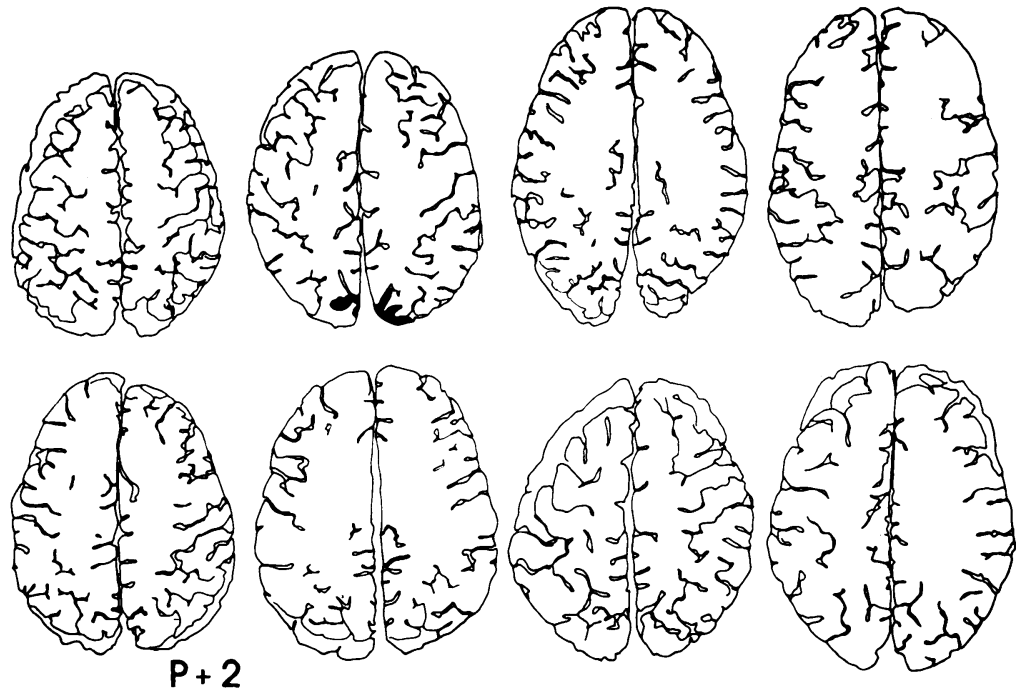

Fig. 7 


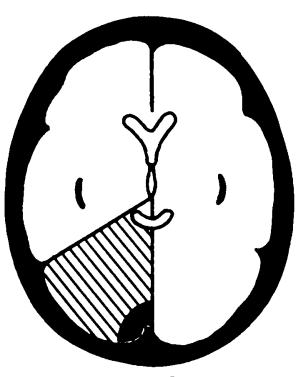

P-1
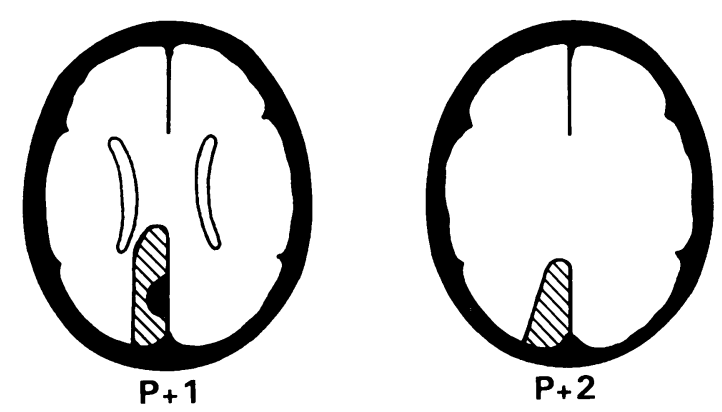

PCA

Cal.c.

Fig. 8 Diagram to show posterior cerebral territory (hatched) and calcarine cortex (solid) in relation to scan levels.

Three other patients had unsuspected infarcts in other parts of the brain. All three were in the frontal region and in one case there was an additional very high left parietal infarct. In none of these cases was cerebral embolism thought to be responsible.

There was a high prevalence of generalised cerebral atrophy. Twelve of the 38 patients had features which led the radiologist to report generalised cerebral atrophy. The difficulty of interpreting these features has already been emphasised (Claveria et al., 1977).

Patients scanned in 1974-75 did not have intravenous enhancement routinely if an infarct was suspected, and thus only patients scanned more recently had an enhanced scan. Of the 14 patients who received intravenous injections of contrast medium, only two showed any enhancement, two and four weeks after the onset of the lesion respectively. Those that did not show enhancement were scanned one month or more after the ictus.

\section{Discussion}

The difficulty of ensuring the correct plane while scanning the patient, and of cutting the cadaver brain slices in a comparable plane has already been discussed. Despite this source of error the study shows the variation in the representation of the visual cortex which the CAT scanner may produce in any one slice. This knowledge is essential before any attempt at correlation of the visual field deficit with the scan findings.

The finding of normal CAT scans in patients with small scotomatous defects was not surprising as this clearly relates to the size of the infarct and the resolution of the scanner. The duration of the infarct is also relevant because at an early stage the involved area may be isodense (Davis et al.. 1975; Masedu et al., 1977). None of our patients was scanned less than two weeks after the onset of the lesion so that the infarcts would be expected to be visible (Yock and Marshall, 1975). Thus it is difficult to account for the normal scan on the patient with a complete hemianopia. Figure 9 shows the visual fields in the two patients with scotomatous defects, one with a normal and the other with an equivocal scan.

The significance of the demonstration of cerebral atrophy on CAT scanning has been discussed by Claveria et al. (1977) without conclusive findings. It is possible that CAT scanning may over-e estimate the presence of cerebral atrophy. The high prevalence of atrophy in our series may also reflect the presence of diffuse cerebrovascular disease.

Almost all the lesions giving rise to isolated hemianopias were situated posteriorly and involved the visual cortex or posterior radiation. In only two patients did the infarct involve only the anterior radiation, sparing the visual cortex entirely (Fig. 14). Both of these patients had quadrantic defects and, to judge from the situation of the infarcts, they may have been within middle rather than posterior cerebral artery territories.

To determine whether a complete hemianopia is associated with a more extensive lesion (appearing in more slices) than a quadrantic or scotomatous defect. the number of involved slices was counted in each category. Complete hemianopias had a mean number of involved slices of 3.0 while upper and lower quadrantanopias combined had a mean of 1.6 slices (Table 2).

There was a difference between those with an upper and those with a lower quadrantanopia. Upper quadrantanopias involved slice $P$ and below except in one case, whereas in lower quadran tanopias none involved slices lower than slice $P$. 
Table 1 CAT scans in infarcts of the posterior visual pathways

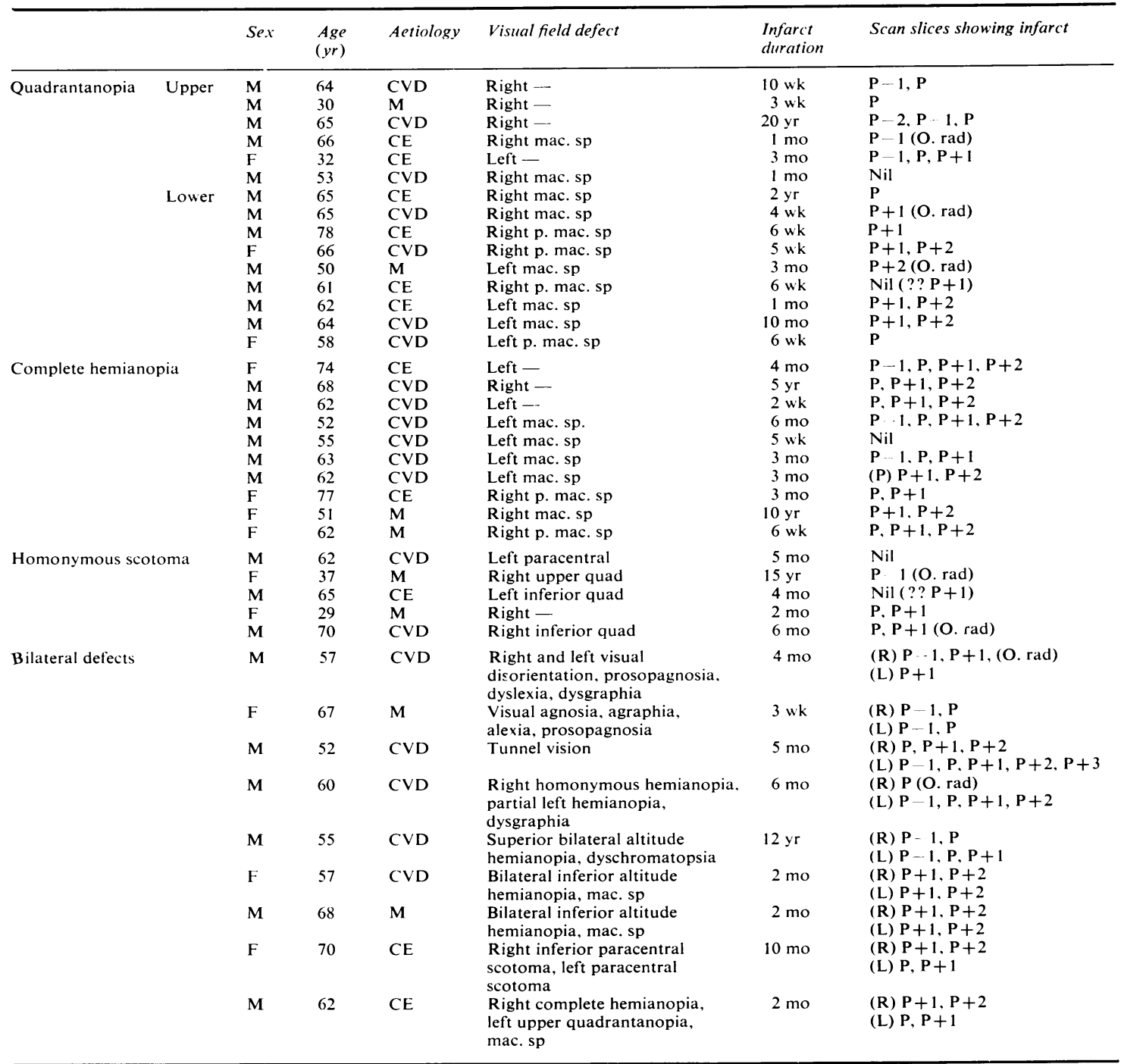

$\mathrm{CVD}=$ cerebrovascular disease $; \mathrm{CE}=$ cerebral embolus; $\mathrm{M}=$ migraine; $\mathrm{P}$. mac. $\mathrm{sp}=$ partial macular sparing; mac. $\mathrm{sp}=$ macular sparing; $\mathrm{O}$. rad $=$ optic radiation.

Table 2 CAT scans showing involvement of individual slices

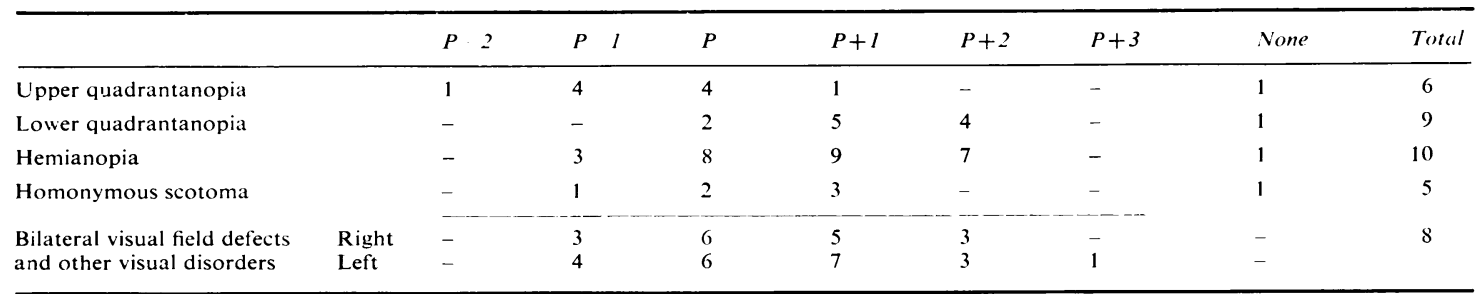

Mean number of slices showing infarct: quadrantanopia $=1.6$ : hemianopia $=3.0$. 

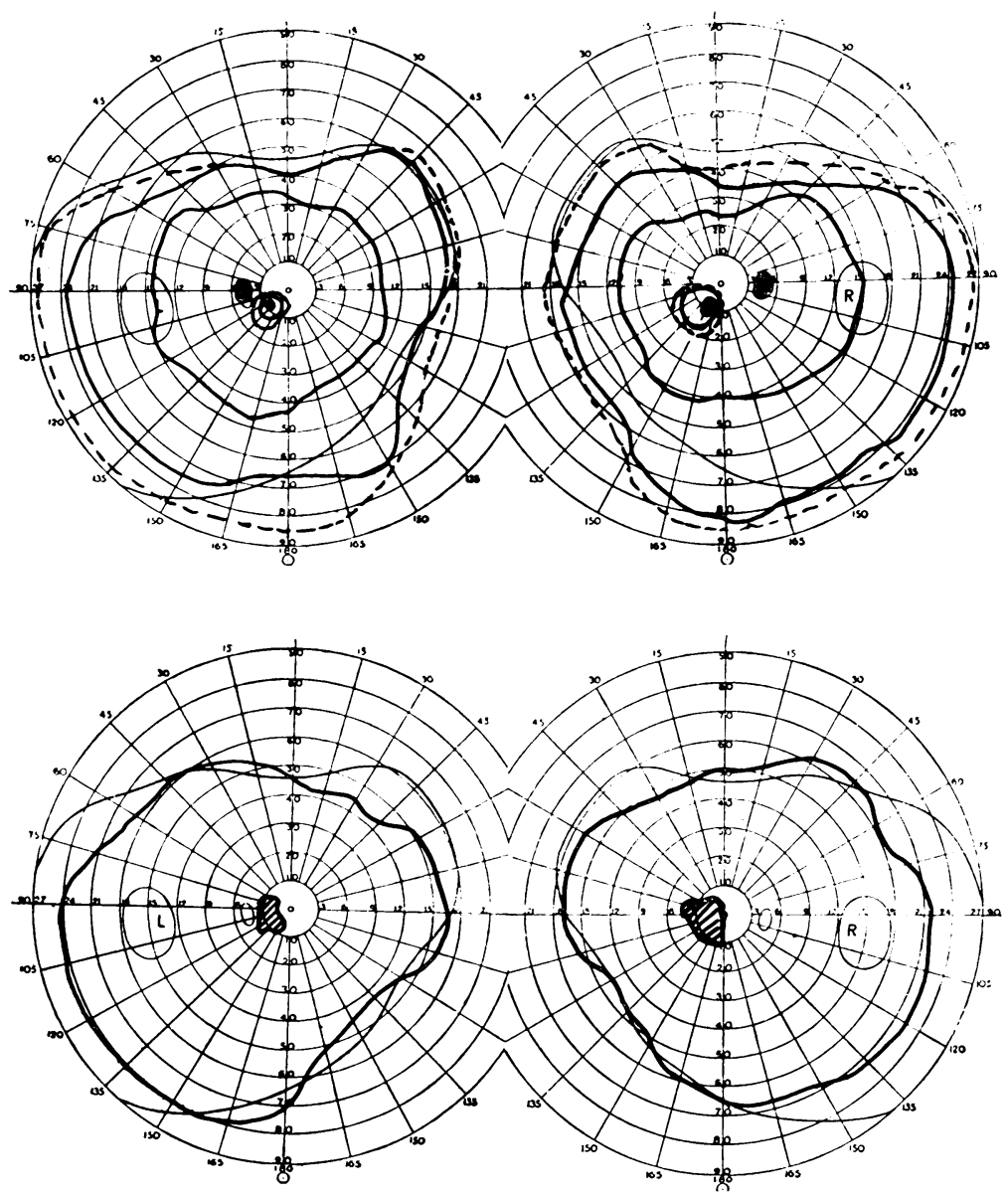

Fig. 9 Visual fields from two patients with homonymous scotomas-one patient had a normal scan and the other an equivocal scan. 


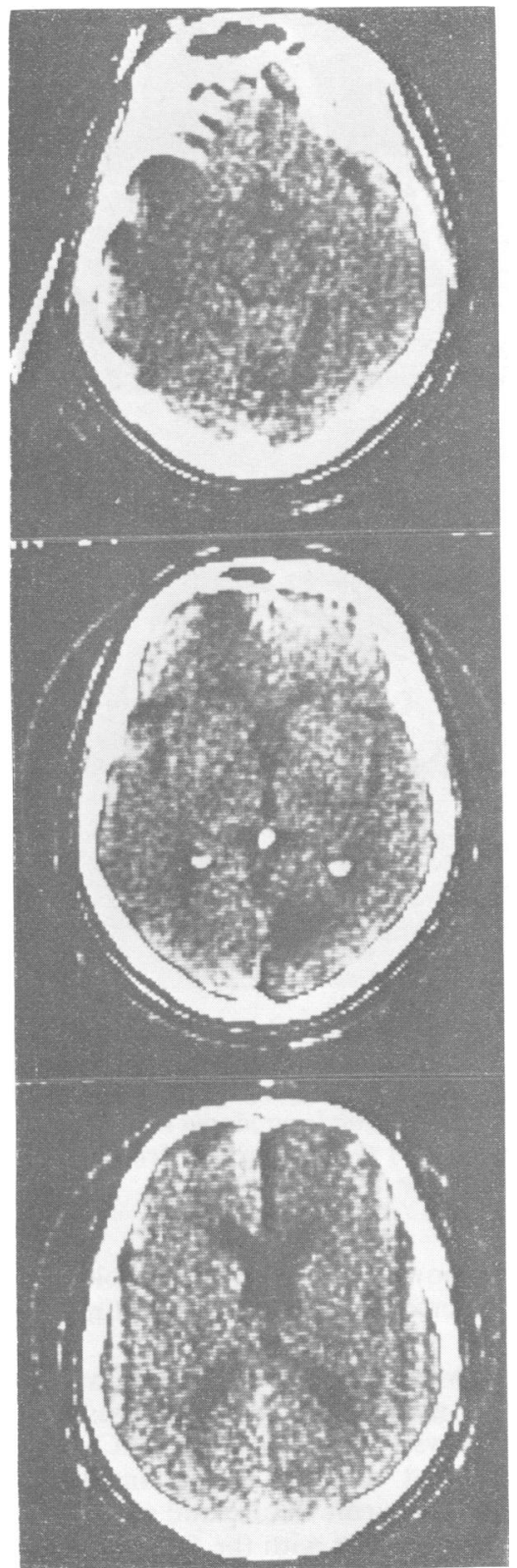

Fig. 10 Scan from a patient with a hemianopia and macular sparing showing preservation of the occipital pole.

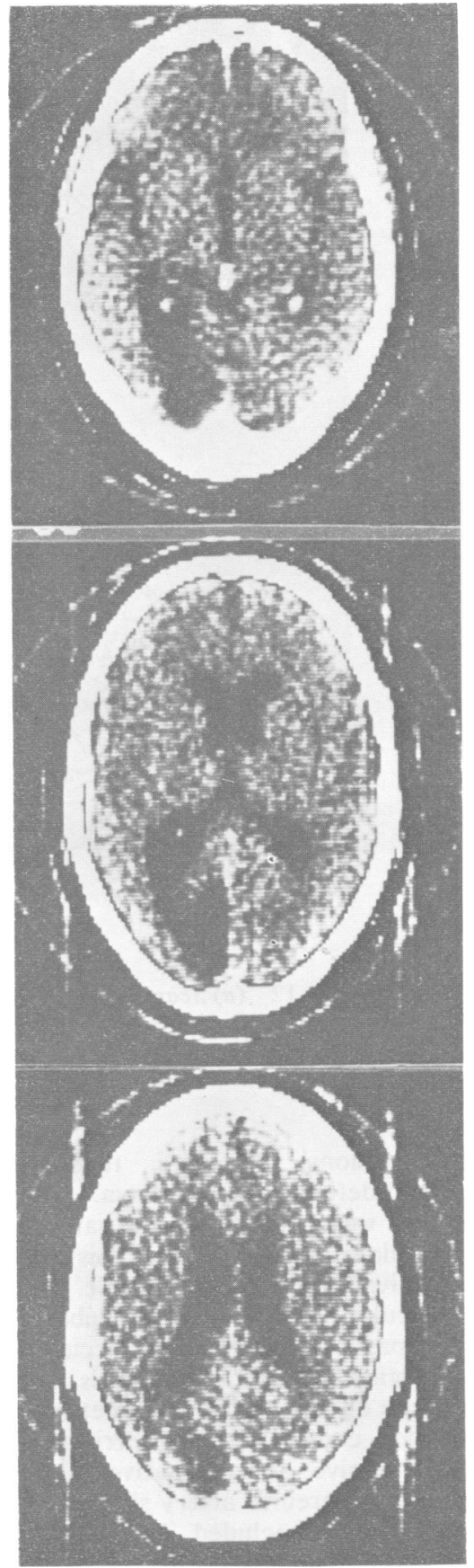

Fig. 11 Scan from a patient with a complete hemianopia showing no preservation of the posterior pole. 


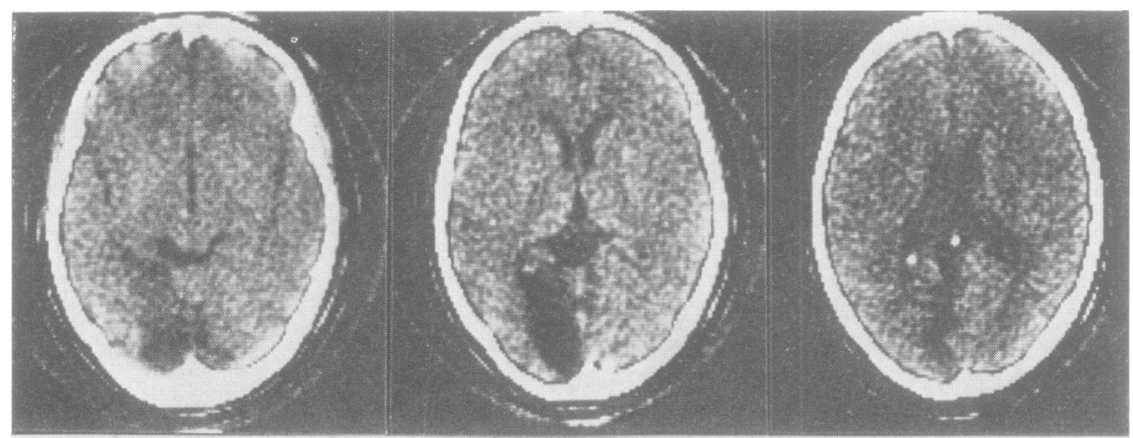

(a)

Fig. 12 (a) Scan showing maximal involvement below the $P$ slice and no preservation of the posterior pole. The visual field defect was a superior quadrantanopia with no macular sparing. (b) Scan showing involvement only of the P slice. The field defect was maximal in the lower quadrant and spared the macula.

Superiorly placed lesions, therefore, tend to produce inferior field defects and vice versa.

Particular interest was taken in those patients who showed a macular sparing to see if this corresponded with survival of the posterior pole. The explanation for macular sparing has been debated extensively in the past. In the case of occipital infarcts the explanation normally invoked is that the posterior pole lies on the border zone between middle and posterior cerebral artery territories. It may, therefore, derive an alternative blood supply from the middle cerebral artery should the posterior cerebral artery be occluded. This is often sufficient to allow the macular cortex and corresponding radiation to survive (Ross Russell, 1973; Kaul et al., 1974). Figure 10 shows the scan from one such patient where preservation of the occipital pole was accompanied by macular spar- ing, while Fig. 11 shows no survival of the pole and in this patient there was complete splitting of central vision.

Correlation between scan findings and visual field defects was good in individual cases. In Fig. 12 the scan shows maximal involvement below the $\mathrm{P}$ slice, consistent with an infarct involving the lower lip of the calcarine fissure and extending to the pole; this is consistent with the field defect.

The nine patients with bilateral lesions were of a similar age and sex distribution to those with unilateral lesions. A cardiac source for embolism was found no more frequently than in those with simple defects. Quadrantic, scotomatous, and complete types of hemianopia were all encountered, and macular sparing was a prominent feature in four patients. In agreement with previous pathological studies (Meadows. 1974) patients with 

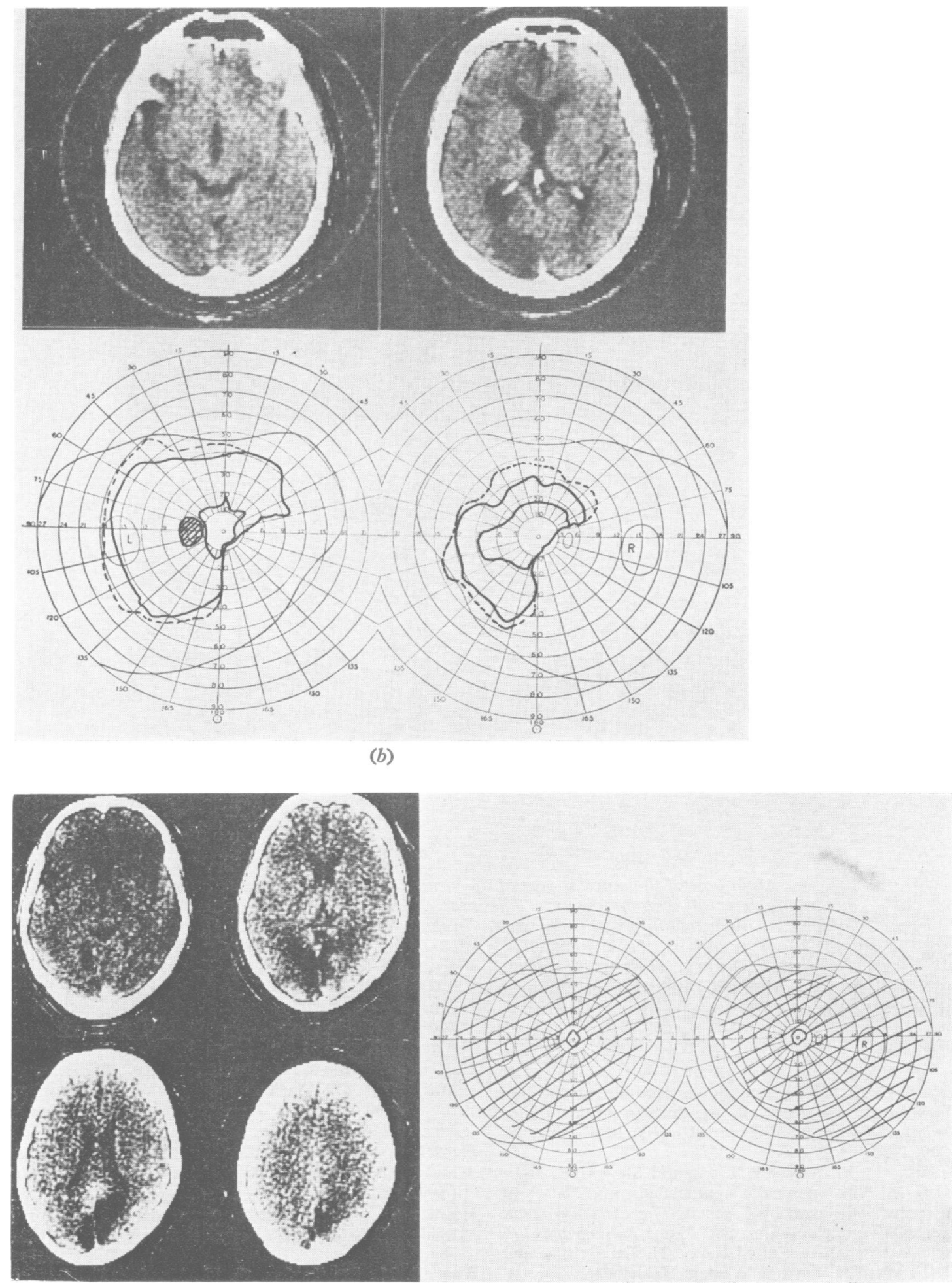

Fig. 13 Scan showing extensive bilateral infarction. The patient had tunnel vision. 

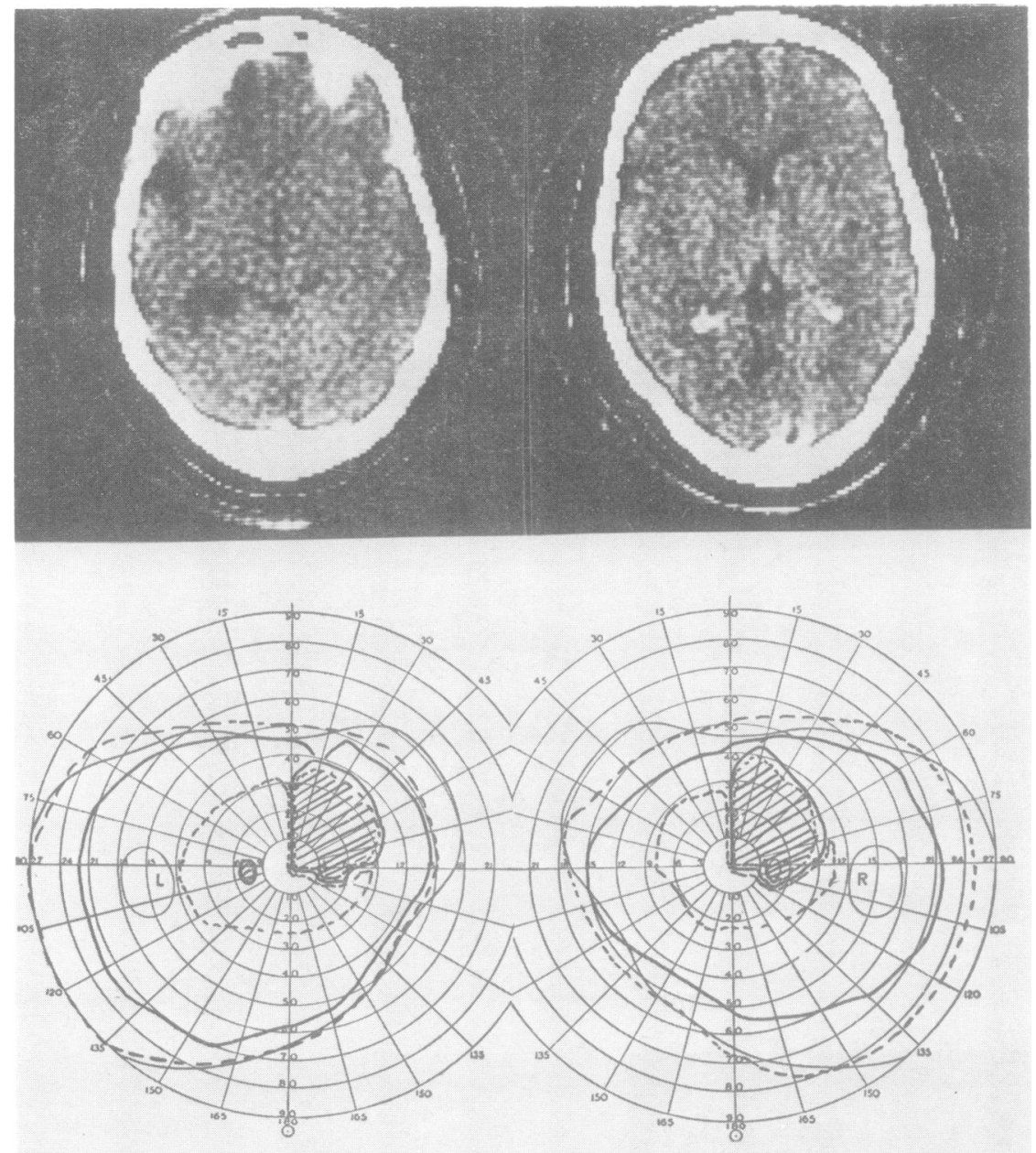

Fig. 14 An infarct of the anterior part of the visual radiation giving rise to a superior quadrantic defect of scotomatous type. The infarct is close to the middle/posterior border zone. A second infarct may be present in the left frontal lobe.

defects such as prosopagnosia, dyschromatopsia, and visual disorientation usually had large bilateral lesions extending outside the striate cortex.

\section{References}

Beevor, C. E. (1909). On the distribution of the different arteries supplying the human brain. Philosophical Transactions of the Royal Society, Series B, 200, 1-55.

Claveria, L. E., Moseley, I. F., and Stevenson, J. F. (1977). The clinical significance of "cerebral atrophy" as shown by CAT. In The First European Seminar on Computerised Axial Tomography in Clinical Practice. Edited by G. H. Du Boulay and I. F. Moseley. Springer-Verlag: Heidelberg.

Davis, K. R., Taveras, J. M., New, P. F. J., Schnur,
J. A., and Robertson, G. H. (1975). Cerebral infarction diagnosis by computerised tomography: analysis and evaluation of findings. American Journal of Roentgenology, Radium Therapy, and Nuclear Medicine, 124, 643-660.

Gawler, J., Bull, J. W. D., Du Boulay, G. H., and Marshall, J. (1975). Computerised axial tomography: the normal EMI scan. Journal of Neurology, Neurosurgery, and Psychiatry, 38, 935-947.

Henschen, S. E. (1926). Zur Anatomie der Schbahn und des Sehzentrums. Albrecht von Graefe's Archiv für Opthalmologie, 117, 403-418.

Holmes, G. (1918). Disturbances of vision by cerebral lesions. British Journal of Ophthalmology, 2, 353384.

Kaul, S. N., Du Boulay, G. H., Kendall, B. E., and Ross Russell, R. W. (1974). Relationship between 
visual field defect and arterial occlusion in the posterior cerebral circulation. Journal of Neurology, Neurosurgery, and Psychiatry, 37, 1022-1030.

McAuley, D. L., and Ross Russell, R. W. (1977). Vascular disease of the visual radiation and cortex. In The First European Seminar on Computerised Axial Tomography in Clinical Practice, pp. 243-248. Edited by G. H. Du Boulay and I. F. Moseley. Springer-Verlag: Heidelberg.

Masdeu, J. C., Azar-Kia, B., and Rubino, F. A. (1977). Evaluation of recent cerebral infarction by computerised tomography. Archives of Neurology (Chicago), 34, 417-421.

Meadows, J. C. (1974). The anatomical basis of prosopagnosia. Journal of Neurology, Neurosurgery, and Psychiatry, 37, 489-501.

Polyak, S. L. (1957). The Vertebrate Visual System. University of Chicago Press: Chicago.

Roberts, M., and Hanaway, J. (1969). Preparation of brain slices for macroscopic study by the copper sulfate-phenol-ferrocyanide technique. Stain Technology, 44, 143-146.

Roberts, P. A., Claveria, L. E., and Moseley, I. F. (1977). Computerised axial tomography and the normal brain. In The First European Seminar on Computerised Axial Tomography in Clinical Practice, pp. 1-16. Edited by G. H. Du Boulay and I. F. Moseley. Springer-Verlag: Heidelberg.

Ross Russell, R. W. (1973). The posterior cerebral circulation. Journal of the Royal College of Physicians of London, 7, 331-346.

Salamon, G. (1973). Atlas of the Arteries of the Human Brain. Second Edition. Sandoz Editions: Paris.

Salamon, G., Lecaque, G., Hall, K., and Corbaz, J. M. (1977). CAT scanning: correlations with vascular and topographical anatomy. In The First European Seminar on Computerised Axial Tomography in Clinical Practice, pp. 17-27. Edited by G. H. Du Boulay and I. F. Moseley. SpringerVerlag: Heidelberg.

Spalding, J. M. K. (1952). Wounds of the visual pathways. Journal of Neurology, Neurosurgery, and Psychiatry, 15, 99-107.

Yock, D. H., and Marshall, W. H. (1975). Recent ischaemic brain infarcts at computed tomography: appearances pre- and post-contrast infusion. Radiology, 117, 599-608. 